\title{
Subcutaneous Emphysema Following Robotic Pancreaticoduodenectomy
}

\author{
Keith M. Cavaness ${ }^{1 *}$ \\ ${ }^{I}$ Department of Surgery, Hepatopancreaticobiliary Surgery, Baylor University Medical Center, Dallas, Texas, \\ 75246, US
}

*Corresponding Author: Keith M. Cavaness, Department of Surgery, Hepatopancreaticobiliary Surgery, Baylor University Medical Center, Dallas, Texas, US. Email: Keith.Cavaness@bswhealth.org

\section{INTRODUCTION}

Pancreaticoduodenectomy has been regarded as one of the most challenging and technically complex abdominal operations. While it is one of the only curative options for pancreatic head tumors, it can have significant morbidities. The complexity of the operation is often a result of the pancreatic anatomy, location and difficult reconstruction to restore gastrointestinal continuity. The introduction of minimally invasive pancreatic surgery has been reported to show reductions in blood loss, analgesic requirements and hospital stay thereby decreasing the overall morbidity. ${ }^{1}$ With the gaining popularity of minimally invasive techniques and the improved functionality of the robotic platform, robotic pancreaticoduodenectomy (RPD) has slowly gained increasing acceptance. While there have been many reports of the successful completion of $\mathrm{RPD}^{2}$, few reports discuss robotic specific morbidities. We would like to present the case of a patient who underwent successful RPD, however due to the prolonged operative time and insufflation; he suffered from diffuse subcutaneous emphysema (SE).

\section{CASE REPORT}

The patient is a 68 year old male who presented to the hospital with nausea, vomiting and jaundice. After a detailed work up he was noted on imaging to have a mass in the head of the pancreas. He underwent endoscopic ultrasound, biopsy and stent placement. The pathology was consistent with a poorly differentiated pancreas ductal adenocarcinoma. The patient was presented at our pancreas multidisciplinary tumor conference and the recommendation was made to proceed with neoadjuvant chemotherapy as part of an institutional trial. He was treated with six cycles of systemic, cytotoxic therapy over a course of 3 months. He tolerated the therapy well without any significant complications. He was restaged and found to have a good response with decrease in the size of the tumor. He was given 4 weeks off treatment to recover in preparation for surgery.

The patient was taken to the operating room and underwent a diagnostic laparoscopy which showed no evidence of metastatic disease and so we proceeded with RPD. After port placement the robot was docked. $\mathrm{CO}_{2}$ was used to maintain a constant pneumoperitoneum at $15 \mathrm{mmHg}$ pressure. The procedure proceeded with kockerization of the duodenum, division of the pancreatic neck, division of the bile duct, division of the proximal jejunum, and then careful dissection of the head and uncinate process away from the portal vein, superior mesenteric vein and superior mesenteric artery. The specimen was removed through a $4 \mathrm{~cm}$ lower transverse incision. The pathologic margins were negative. The extraction incision was partially closed around a $10 \mathrm{~mm}$ port that was used for the remainder of the case. The operative field was hemostatic and so we proceeded with reconstruction. An end-to-side pancreaticojejunostomy was completed in a two layer fashion using the Blumgart technique. An end-to-side hepaticojejunostomy was then completed followed by a stapled gastrojejunostomy. A feeding jejunostomy was placed $30 \mathrm{~cm}$ distal to the gastrojejunostomy for postoperative nutrition. Two drains were placed anterior and posterior to our biliary and pancreatic anastomoses. The incisions were then closed and the patient was taken to the Intensive Care Unit in stable condition. The operative time was 580 minutes. Blood loss was $250 \mathrm{ml}$. 
The patient remained hemodynamically stable throughout the procedure.

In the immediate postoperative period the patient was noted to have diffuse SE extending from the mid-thighs up to the face. A CT chest was completed showing no injury to the airway and bilateral apical pneumothoraces. The patient was hemodynamically stable however he continued to require mechanical ventilation and so the decision was made to place bilateral tube thoracostomies to prevent worsening of the pneumothoraces from positive pressure ventilation. The chest tubes were placed without difficulty. The patient was extubated the morning of post-operative day 1 . The chest tubes were removed on post-operative day 3 . The SE resolved on postoperative day \#4. The patient made an uneventful recovery and was discharged home on post-operative day 7 .

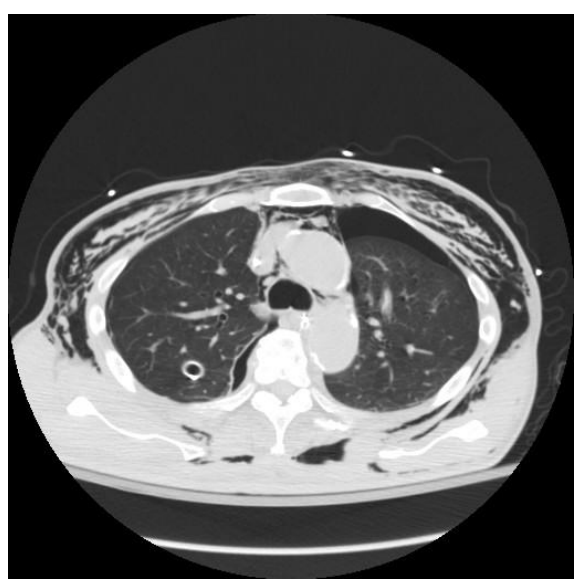

Figure1.CT chest showing subcutaneous emphysema

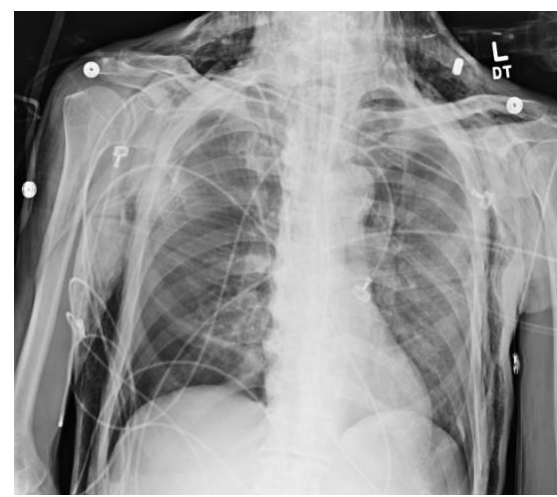

Figure2. Chest $x$-ray showing subcutaneous emphysema

\section{DISCUSSION}

Establishing pneumoperitoneum in laparoscopic surgery is vital. ${ }^{3}$ Traditionally, carbon dioxide $\left(\mathrm{CO}_{2}\right)$ has been used for insufflation due to its rapid absorption and ease of use. Although insufflations with carbon dioxide have been considered safe, there are risks of developing various complications including SE, hypercarbia, pneumothorax, pneumomediastinum and carbon dioxide embolism.

SE results from the introduction of gas into the soft tissue. Various studies have reported SE in the setting of laparoscopic surgery where forced air insufflation creates a potential space in the subcutaneous tissue resulting in diffuse air pockets and crepitus. Factors that may increase the risk include; operative time greater than 200 minutes, use of more than 5 ports and older age. ${ }^{4}$ Lee et al suggested that prolonged increase in intraabdominal pressure during laparoscopy can also lead to SE. ${ }^{5}$ The incidence of SE has increased as laparoscopic and robotic operations have become more common.

In our case, the specimen was removed at the mid-point of the operation in order to check surgical margins. The extraction incision was then partially closed. After the margins were cleared, we proceeded with the complicated and time consuming reconstruction. The extraction incision was used for the remainder of the case with continuing positive pressure insufflation. We feel that this allowed introduction of $\mathrm{CO}_{2}$ into the subcutaneous tissues creating SE. While this did not cause any life threatening complications, it did create bilateral pneumothoraces which required treatment. The patient recovered well and was discharged home one week after surgery. The patient did not suffer any long term sequela.

As complicated robotic surgery becomes more common, operative times will likely increase. Complications associated with $\mathrm{CO}_{2}$ insufflation should be evaluated in the immediate postoperative period. SE should be evaluated closely in the perioperative period to prevent serious complications. In this age of advanced minimally invasive surgery it is critical that we understand the dynamics of the robotic platform and the complications that can occur in order to reduce morbidity and improve patient care.

\section{REFERENCES}

[1] Venkat R, Edil BH, Schulick RD, Lidor AO, Makary MA, Wolfgang CL. Laparoscopic distal pancreatectomy is associated with significantly less overall morbidity compared to the open technique: a systematic review and metaanalysis. Ann Surg. 2012; 255(6):1048-1059. doi:10.1097/SLA.0b013e318251ee09.

[2] Giulianotti PC, Sbrana F, Bianco FM, et al. Robot-assisted laparoscopic pancreatic surgery: single-surgeon experience. Surg Endosc. 2010; 24(7):1646-1657. doi: 10.1007/s00464-0090825-4. 
[3] Cheng Y, Lu J, Xiong X, et al. Gases for establishing pneumoperitoneum during laparoscopic abdominal surgery. Cochrane database Syst Rev. 2013;(1):CD009569. doi:10.1002/14651858.CD009569.pub2.

[4] Murdock CM, Wolff AJ, Van Geem T. Risk factors for hypercarbia, subcutaneous emphysema, pneumothorax, and pneumomediastinum during laparoscopy. Obstet Gynecol. 2000; 95(5):704-709.

[5] Lee DW, Kim MJ, Lee YK, Lee HN. Does intraabdominal pressure affect development of subcutaneous emphysema at gynecologic laparoscopy? J Minim Invasive Gynecol. 2011; 18(6):761-765. doi:10.1016/j.jmig.2011.08.006.

Citation: Keith M. Cavaness, Subcutaneous Emphysema Following Robotic Pancreaticoduodenectomy. ARC Journal of Surgery. 2017; 3(3):14-16. doi: dx.doi.org/ 10.20431/2455-572X. 0303005.

Copyright: () 2017 Authors. This is an open-access article distributed under the terms of the Creative Commons Attribution License, which permits unrestricted use, distribution, and reproduction in any medium, provided the original author and source are credited. 